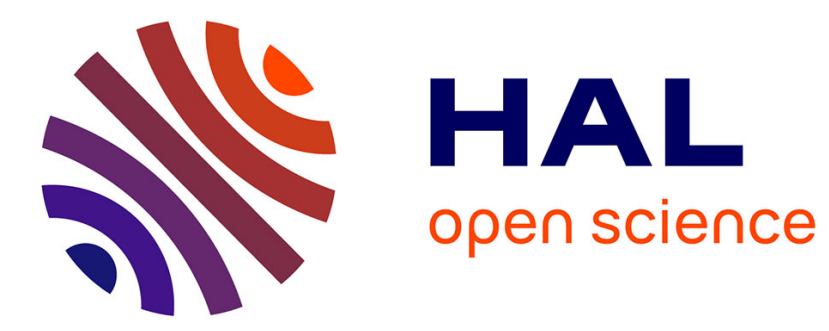

\title{
Cavitation erosion: Using the target material as a pressure sensor
}

\author{
Samir Chandra Roy, Jean-Pierre Franc, Marc C. Fivel
}

\section{To cite this version:}

Samir Chandra Roy, Jean-Pierre Franc, Marc C. Fivel. Cavitation erosion: Using the target material as a pressure sensor. Journal of Applied Physics, 2015, 118 (16), 10.1063/1.4934747 . hal-01223687

\section{HAL Id: hal-01223687 \\ https://hal.science/hal-01223687}

Submitted on 5 May 2020

HAL is a multi-disciplinary open access archive for the deposit and dissemination of scientific research documents, whether they are published or not. The documents may come from teaching and research institutions in France or abroad, or from public or private research centers.
L'archive ouverte pluridisciplinaire HAL, est destinée au dépôt et à la diffusion de documents scientifiques de niveau recherche, publiés ou non, émanant des établissements d'enseignement et de recherche français ou étrangers, des laboratoires publics ou privés. 


\title{
Cavitation erosion: Using the target material as a pressure sensor
}

\author{
Samir Chandra Roy, ${ }^{1, \text { a) }}$ Jean-Pierre Franc, ${ }^{2}$ and Marc Fivel ${ }^{1}$ \\ ${ }^{1}$ Science and Engineering of Materials and Processes (SIMaP-GPM2), University Grenoble Alpes/CNRS, \\ F-38000 Grenoble, France \\ ${ }^{2}$ Laboratory of Geophysical and Industrial Flows (LEGI), University Grenoble Alpes/CNRS, \\ F-38000 Grenoble, France
}

Numerical prediction of mass loss due to cavitation erosion requires the knowledge of the hydrodynamic impact loads generated by cavitation bubble collapses. Experimental measurements of such impact loads using conventional pressure sensors are not reliable (if not impossible) due to the micron size and the very small duration of the loading. In this paper, a new method to estimate these loading conditions is proposed based on cavitation pitting tests and an iterative inverse finite element modeling. The principle of the method is as follows. First, numerous pits corresponding to localized plastically deformed regions are identified from a cavitation test performed in a dedicated tunnel. Then each pit is numerically reproduced by finite element simulations of the material response to a representative Gaussian pressure field supposed to mimic a single bubble collapse. This gives the size and pressure distribution of the bubble impacts. The prime objective of this study is to find out if the target material itself could be used as a pressure sensor or not, i.e., if the cavitation pits left on the surface of the tested specimen could provide the characteristics of the cavitating flow in terms of pressure fields independently of the target material. Pitting tests were done on three materials, namely, 7075 Aluminum alloy (Al-7075), 2205 duplex stainless steel (A-2205), and Nickel-Aluminum Bronze (NAB) at three different flow conditions and the impact loads have been estimated for each identified pit. Very interestingly, a statistical analysis shows that the estimated impact loads are material independent at all flow conditions, provided the material properties are characterized properly. It is also shown that for some materials, the constitutive parameters obtained from compression tests are not satisfactory.

\section{INTRODUCTION}

Cavitation erosion occurs in fluid machineries, pumps, pipes, ship propellers, valves, and so on ${ }^{1}$ mainly due to rapid fluctuation of fluid pressure associated with high velocity. Rapid fluctuation of pressure causes the formation of cavitation bubbles which subsequently collapse, leading to the formation of high velocity micro-jets and/or shock waves that produce a high impact on the solid wall. ${ }^{2,3}$ Life prediction of such components depends on the prediction of the cavitation erosion rate which requires the estimation of the impact loads generated by cavitation bubble collapse ${ }^{4}$ and has been a challenging issue to date.

Direct measurement of impact loads using commercial piezoelectric transducer, ${ }^{5}$ ceramics pressure transducers, ${ }^{4}$ pressure transducers made of Polyvinylidene Fluoride (PVDF) piezoelectric films, ${ }^{6}$ and crystals of magnesium oxide along with piezoceramic, ${ }^{7}$ has been the common practice. However these transducers may not provide reliable measurements of the impact loads mainly because of their large sensitive surface area compared to the micron size of the hydrodynamic impact loads. Moreover, the load is not uniformly applied on to the transducer surface, which is the basic assumption required to estimate the impact stress

\footnotetext{
${ }^{\text {a)} E l e c t r o n i c ~ a d d r e s s e s: ~ r o y s a m . n i t a @ g m a i l . c o m ~ a n d ~ s a m i r-c h a n d r a . r o y @ s i-~}$ map.grenoble-inp.fr
}

(MPa) corresponding to the transducer output signal expressed in force unit $(\mathrm{N}$ or $\mu \mathrm{N})$. Finally, these transducers have limited natural frequency and rise time which affect the measurements.

Numerical approaches may also be used to compute the pressure pulses due to the collapse of a single bubble or bubble clusters that may develop in real flows, such as the flow around a cavitating foil or in a cavitating hydraulic device (see, e.g., Refs. 8 and 9), but there are also limitations arising from the fluid side in defining air contents, initial bubble sizes, and their standoff distances, local pressure gradient, possible bubble interactions, etc., into the numerical simulations to be relevant to the real scenario.

In order to avoid these measurement difficulties, the authors have recently proposed a method in Ref. 10 to estimate the hydrodynamic impact loads. The method consists of pitting tests as introduced by Knapp ${ }^{11,12}$ and iterative inverse finite element (FE) simulations. The idea behind pitting tests is that each pit is a localized plastically deformed region and is the signature of a single bubble collapse. The principle of the method ${ }^{10}$ is to numerically reproduce the experimental cavitation pits (characterized by pit depth, $h_{P}$ and diameter, $d_{P}$ measured at mid-depth) by FE modeling of the material response to a representative Gaussian pressure field (characterized by peak stress, $\sigma_{H}$, and radial extent, $d_{H}$ ). We have shown that a Gaussian type 
of pressure field could be considered relevant to the cavitation impact loading. The material behavior is modeled as elasto-plastic isotropic hardening. In the static simulations presented in Ref. 10, the dynamic behavior of cavitation pitting is taken into account by extrapolating the material properties to a high strain rate of $10^{6} \mathrm{~s}^{-1}$ that corresponds to an impact duration of a microsecond. ${ }^{13}$ Dynamic simulations were also conducted in order to investigate the limitations of such a static approach and discussed in Ref. 13. More details regarding the material model and dynamic simulations are discussed in Sec. II.

Note that a similar Gaussian pressure field is used by other authors ${ }^{14,15}$ as well for cavitation pitting simulation, but very few conducted inverse modelling in order to access the flow parameters. Pohl et al. ${ }^{16}$ have done a similar inverse analysis by comparing the simulated and experimental pit geometry, however, by using a bell-shape pressure profile. Moreover, it was not shown whether similar pit geometry can be obtained by other sets of pressure field parameters or not. They have used the simplex method for the optimization step that would be very time consuming to implement on a large number of pit data. Finally, the authors did not take into account the strain rate sensitivity in the material modelling.

In this paper, the inverse FE method proposed in Ref. 10 is applied on three materials; namely, 7075 Aluminum alloy (Al-7075), 2205 duplex stainless steel (A-2205), and NickelAluminum Bronze (NAB), to estimate the impact loads and their radial extent. Pitting tests were done (testing method is explained in details in Refs. 17 and 18) on these three materials at different cavitation flow conditions as discussed in Sec. III A.

Now, if the material properties are properly characterized, the estimated impact loads should be material independent or, in other words, the estimated impact loads should characterize the same flow condition irrespective of the material being used to capture them. Thus the characterization of the material properties is key to the accuracy of the estimated impact loads, and a special effort is made in this paper to find out the most appropriate way of material characterization with respect to cavitation erosion phenomena.

Although the nanoindentation test is used by many authors $^{16,19,20}$ to characterize the deformation behavior of materials under cavitation impact, the strain rate involved in cavitation is significantly higher (typically $10^{3}-10^{4} \mathrm{~s}^{-1}$ or even more ${ }^{21}$ ) than those achievable in conventional indentation devices. In the current study, compression and nanoindentation tests have been supported by split Hopkinson pressure bar (SHPB) tests in order to characterize the dynamic material behavior relevant to cavitation loading conditions. A special emphasis is put on the importance of local characterization (as obtained by a nanoindentation test) of heterogeneous material such as Al-7075 compared to the bulk mechanical characterization (as obtained by a compression test).

Thus, this paper gives an overall view on the usability of the target material as a sensor, characterization of material parameters, and the difficulties associated with such methods as well. In Sec. II, characterization of the material properties is discussed in detail. In Sec. III, the main results obtained by the inverse FE method are shown and Sec. IV describes the microstructure of the three materials considered in this study in order to discuss potential differences between different testing techniques.

\section{MATERIAL CHARACTERIZATION}

\section{A. Material and constitutive law}

Three materials, 7075 Aluminum alloy (Al-7075), 2205 duplex stainless steel (A-2205) and Nickel-Aluminum Bronze (NAB), have been considered for the current study. The Johnson-Cook (JC) plasticity model in the form given by Eq. (1) (without considering the thermal softening part) is used to characterize the hardening behavior of the materials (see Ref. 22 for more details about the JC plasticity model)

$$
\bar{\sigma}=\left(\sigma_{y}+K \varepsilon_{p}^{n}\right)\left(1+C \ln \frac{\dot{\varepsilon}_{p}}{\dot{\varepsilon}_{0}}\right) .
$$

Here, $\varepsilon_{p}$ is the equivalent plastic strain, $\dot{\varepsilon}_{p}$ is the equivalent plastic strain rate, and $\dot{\varepsilon}_{0}$ is the reference strain rate (generally taken as $\dot{\varepsilon}_{0}=1 \mathrm{~s}^{-1}$ ) at which the yield strength $\sigma_{y}$, strength coefficient $K$, and strain hardening exponent $n$ have been estimated. $C$ is the strain rate sensitivity parameter. At the reference plastic strain rate $\dot{\varepsilon}_{p}=\dot{\varepsilon}_{0}$, Eq. (1) becomes a simple Ramberg-Osgood type equation where the hardening is a function of plastic strain $\varepsilon_{p}$ only.

Generally, nanoindentation is preferred to characterize the material behavior in cavitation pitting, ${ }^{16,20}$ whereas the compression or tension test is commonly used to verify the material constitutive parameters obtained by the nanoindentation test. ${ }^{23,24}$ Hence in this study, both compression and nanoindentation tests were done to obtain the constitutive parameters. Young's modulus $(E)$, Poisson's ratio $(\nu)$, and density $(\rho)$ of the materials are considered to be the same in both compression and nanoindentation, and are given in Table I. Reference strain rate $\left(\dot{\varepsilon}_{0}\right)$ is considered to be $1.0 \mathrm{~s}^{-1}$ for the compression tests and $0.05 \mathrm{~s}^{-1}$ for the nanoindentation tests.

\section{B. Material properties obtained by compression tests}

Cylindrical specimens of equal length and diameter of $8 \mathrm{~mm}$ have been used. Compression tests were done on the three materials at reference strain rate $1.0 \mathrm{~s}^{-1}$ (shown in Figure 1) and $\sigma_{y}, K$, and $n$ were estimated as shown in Table I by fitting Eq. (1) with $C=0$. The fitted curves are shown in Figure 1 as thin black lines.

TABLE I. Material density, Poisson's ratio, Young's modulus and compressive properties at strain rate $1.0 \mathrm{~s}^{-1}$.

\begin{tabular}{lcccccc}
\hline \hline Material & $\sigma_{y}(\mathrm{MPa})$ & $K(\mathrm{MPa})$ & $n$ & $E(\mathrm{GPa})$ & $\nu$ & $\rho\left(\mathrm{kg} / \mathrm{m}^{3}\right)$ \\
\hline Al-7075 & 500 & 312 & 0.29 & 71.9 & 0.33 & 2810 \\
A-2205 & 560 & 917 & 0.51 & 186 & 0.30 & 7805 \\
NAB & 300 & 1205 & 0.56 & 122 & 0.32 & 7580 \\
\hline \hline
\end{tabular}




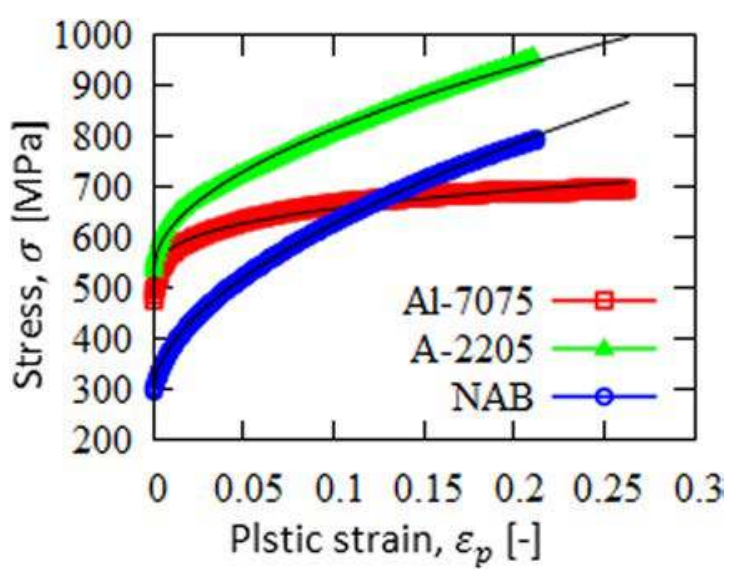

FIG. 1. Compression stress-strain curves (thick lines) of Al-7075, A-2205 and NAB obtained at strain rate $1.0 \mathrm{~s}^{-1}$ using a servo-hydraulic controlled testing machine and fitted curves (thin lines) obtained using Eq. (1) with $C=0$.

\section{Strain rate sensitivity}

In cavitation pitting, the target material deforms compressively in a confined region at a very high strain rate. Thus inertial and strain rate effect become important and should be taken into account in the simulations. Though it is debatable, typical impact duration in cavitation pitting is believed to be on the order of a few microseconds, ${ }^{4,5}$ and recently authors ${ }^{13,15}$ have shown that if the impact duration is greater than $1 \mu$ s then the inertial effect becomes insignificant. Thus we could reliably use static FE analysis for simulating cavitation impact behavior of materials, provided strain rate effect is considered. Although not published, during our earlier study ${ }^{10}$ it was found that practically the strain may exceed $30 \%$ to form a cavitation pit. Of course strain is not uniform throughout the deformed region. Thus if we consider that $30 \%$ of strain occurs in cavitation pitting in $1 \mu \mathrm{s}$ or less time, the strain rate would be $3 \times 10^{5} \mathrm{~s}^{-1}$ or more. In our study to take into account the strain rate effect in cavitation pitting, the material properties were extrapolated to a strain rate of $10^{6} \mathrm{~s}^{-1}$ by using the JC plasticity model (Eq. (1)). In reality the strain rate will depend on the impact duration which again depends on bubble size, pressure gradient, and standoff distance ${ }^{14}$ However, it should be mentioned that, to date, it is not experimentally possible to know the impact duration corresponding to each pit resulting from impact loading of cavitation bubble collapse. Thus, for the inverse FE method ${ }^{10}$ to estimate impact load parameters from cavitation pit parameters, complete dynamic explicit analysis including strain rate sensitivity where impact duration is an essential input parameter, is out of the scope in the present context. From a statistical point of view, static FE analysis with the extrapolated material properties seems to be a reliable option.

To verify such a high strain rate used in the inverse FE method, dynamic explicit simulations of cavitation pitting with the same material model (Eq. (1)), which now additionally takes into account the inertial and strain rate effects, were done on these three materials. For that purpose, the Gaussian pressure field in Ref. 10 is modified as given in Eq. (2) for dynamic simulation to take into account the temporal evolution of stress. It was observed that for characteristic impact duration $t_{H} \approx 1 \mu \mathrm{s}$, the maximum principle strain rates in all three materials were close to $10^{6} \mathrm{~s}^{-1}$

$$
\left.\sigma=\sigma_{H} \exp -\left(\frac{2 r}{d_{H}}\right)^{2}\right) \exp \left(-\left(\frac{t-t_{\max }}{t_{H}}\right)^{2}\right) .
$$

Here, $t$ represents the time, $t_{\max }$ is the time when $\sigma=\sigma_{H}$, and $t_{H}$ is the characteristic impact duration in a similar sense of $d_{H}$.

In order to estimate the strain rate sensitivity, additional compression tests complemented by SHPB tests were done on the three materials at strain rates ranging from 0.001 to $\sim 1500 \mathrm{~s}^{-1}$. For SHPB tests cylindrical specimens of equal length and diameter of $8 \mathrm{~mm}$ have also been used. SHPB tests were done particularly for the highest strain rates, $>10 \mathrm{~s}^{-1}$ (see Ref. 25 for details of experimental setup and analysis procedure for the SHPB test).

The strain rate sensitivity parameter $C$ was estimated by fitting Eq. (3) to the experimental data as shown in Figure 2 in the case of A-2205. To avoid thermal softening $C$ was estimated at a low amount of plastic strain $(<2 \%)$. As can be seen in Figure 2 the values of $C$ estimated at $0.5 \%$ and $1.5 \%$ of plastic strains are almost the same

$$
R=\frac{\bar{\sigma}}{\sigma_{y}+K \varepsilon_{p}^{n}}=1+C \ln \frac{\dot{\varepsilon}_{p}}{\dot{\varepsilon}_{0}} .
$$

In Eq. (3), $R$ represents the stress ratio at strain rate $\dot{\varepsilon}_{p}$ with respect to $\dot{\varepsilon}_{0}$. As expected for metals, the plot of $R$ versus strain rate follows almost a linear relationship in a semi-log plot. Values of $C$ were estimated to be $0.0068,0.031$, and 0.0119 for Al-7075, A-2205, and NAB, respectively. It can be seen that A-2205 has the maximum strain rate sensitivity whereas Al-7075 has the minimum strain rate sensitivity.

Stress-strain curves at strain rate $10^{6} \mathrm{~s}^{-1}$ (as shown in Figure 3) were constructed from the reference curves in Figure 1 by using the estimated strain rate sensitivity in Eq. (1). These stress-strain curves are now used for the FE simulations of cavitation pitting to estimate the hydrodynamic

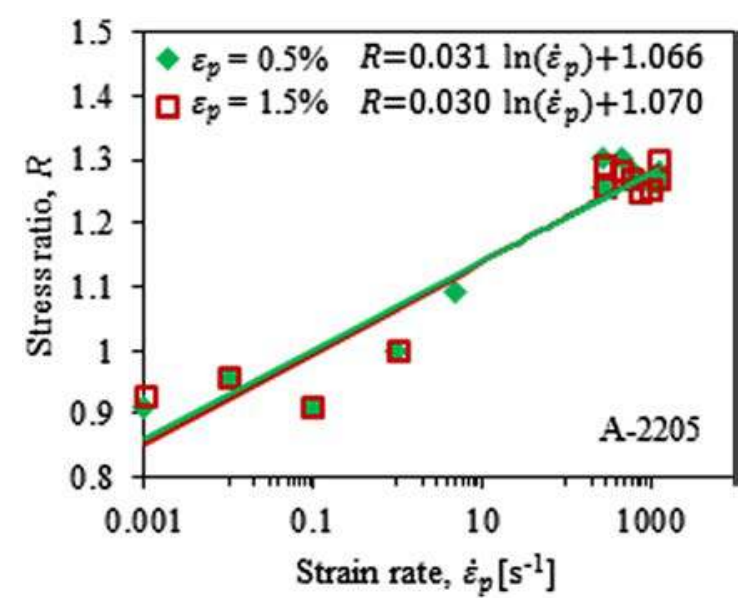

FIG. 2. Stress ratio versus strain rate plot on semi-log scale shows almost linear behavior for A-2205 material. Strain rate sensitivity $C$ is estimated at $0.5 \%$ and $1.5 \%$ of plastic strains. 


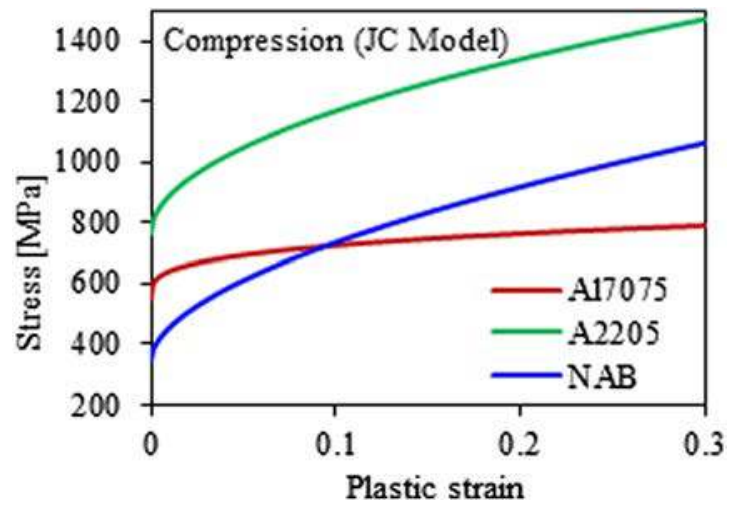

FIG. 3. Stress-strain curves extrapolated to a strain rate of $10^{6} \mathrm{~s}^{-1}$ using the Johnson-Cook plasticity model (Eq. (1)). Reference stress-strain curves were obtained by compression tests.

impact parameters $\sigma_{H}$ and $d_{H}$ from the cavitation pit geometry by using the inverse FE method.

\section{Material properties obtained by nanoindentation tests}

Because of a similarity in material deformation behavior during nanoindentation and cavitation pitting, nanoindentation tests were conducted on these three materials at a strain rate of $0.05 \mathrm{~s}^{-1}$ using a spherical diamond (Young's modulus, $E=1141 \mathrm{GPa}$, and Poisson's ratio, $\nu=0.07$ ) indenter of nominal radius, $R=9.46 \mu \mathrm{m}$. Sample preparation consists of mechanical polishing phases using sandpaper by gradually reducing grit size until $8.4 \mu \mathrm{m}$ (grade P2500), followed by polishing with diamond paste, gradually reducing the size from 6 to $1 \mu \mathrm{m}$, and finally by using colloidal silica of $0.03 \mu \mathrm{m}$ size.

Material properties were obtained by FE simulations of nanoindentation with arbitrarily defined material parameters while comparing the simulated and experimental load displacement curves. The arbitrarily defined material properties also include compression test data. Nowadays this is a very popular practice to obtain nanoindentation material properties by an inverse numerical method using FE simulations. $^{26,27}$ The background of the method adopted here is as proposed by Moussa et al. ${ }^{26}$ Nanoindentation simulations were done in the FE code ABAQUS using a 2D axisymmetric model with four node quadratic elements (CAX4R). Finite sliding, node-to-surface contact formulation with a coefficient of friction 0.1 between the indenter and sample material, is used to model the contact. The indenter was modeled as an elastic material, whereas the test material was modeled as an isotropic elasto-plastic material. ABAQUS static simulations were conducted.

It was found that the nanoindentation simulations performed with the material constitutive parameters estimated from the compression test conducted at a strain rate of $0.05 \mathrm{~s}^{-1}$ yielded almost the same load-displacement curve for A-2205 and NAB, as shown in Figures 4(a) and 4(b), respectively, whereas for Al-7075 the discrepancy is significant, see Figure 4(c). Thus it was concluded that for A-2205 and NAB, the compression test best describes the nanoindentation behavior, which is not true for Al-7075. One important fact in Figure 4(c) is that the simulated material behavior is harder than the real nanoindentation behavior of the material. The reason for this discrepancy for Al-7075 is discussed in detail in Sec. IV.

For Al-7075, real material properties were obtained by varying the constitutive parameters $\left(\sigma_{y}, K\right.$, and $\left.n\right)$ in the FE simulations in order to fit the experimental load-displacement curve. A large number of FE simulations were done for $\sigma_{y}=200-500 \mathrm{MPa}, K=150-450 \mathrm{MPa}$, and $n=0.08-0.4$. The root mean square error between simulated and experimental
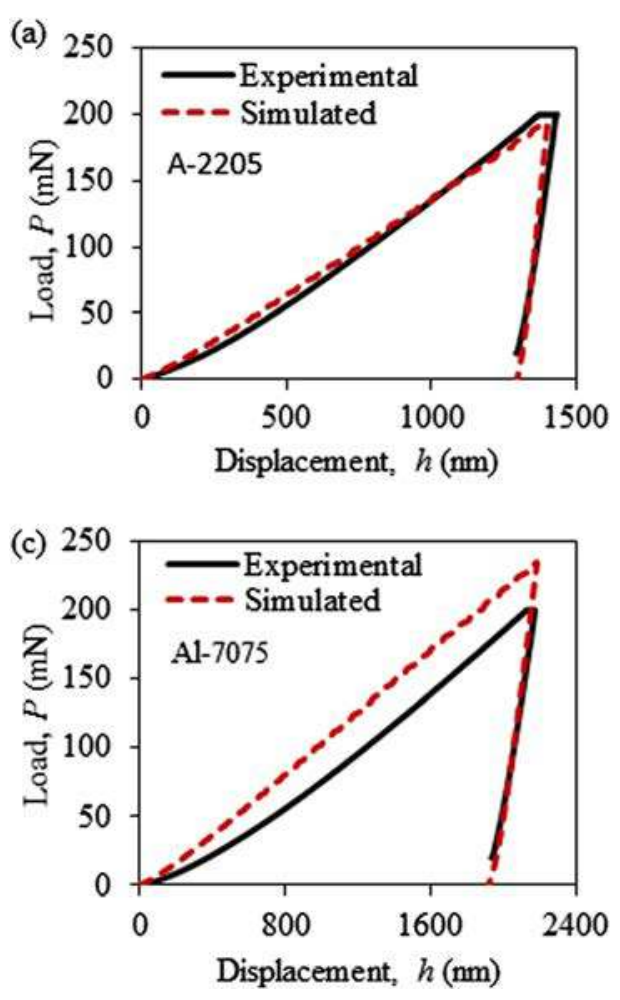
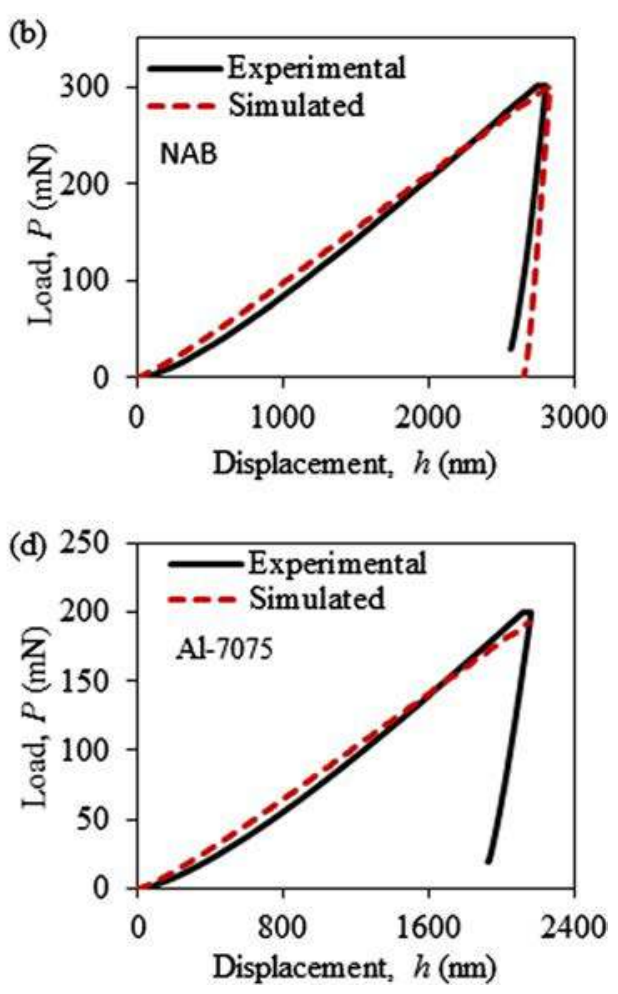

FIG. 4. Comparison of experimental and simulated nanoindentation loaddisplacement curves for all three materials. The material properties obtained by compression tests (Table I) gave the acceptable solution for (a) A-2205 and (b) NAB but not for (c) Al-7075. (d) The acceptable solution for Al-7075 is obtained by manually optimizing the constitutive parameters as discussed in Sec. II D. 
load-displacement curves was estimated as presented in Ref. 26. Unlike what the authors found in Ref. 26, we did not get a unique solution for the constitutive parameters, but several sets of constitutive parameters yielded almost the same loaddisplacement curve for which the errors were smaller than $8 \mathrm{mN}$ and considered as acceptable. An example is shown in Figure 4(d). All the stress-strain curves for which errors are smaller than $8 \mathrm{mN}$ are shown in Figure 5 by dotted lines. An average curve of all of these curves (dashed line) is taken as the characteristic of the material behavior. As can be seen, the nanoindentation strength of the material is significantly reduced compared to the compressive strength (solid line).

The estimated material properties that are characteristic of nanoindentation behavior at a strain rate of $0.05 \mathrm{~s}^{-1}$ are given in Table II for all three materials. The comparison of Tables I and II confirms that the material properties derived from compression and nanoindentation tests are almost identical for both A-2205 and NAB (the small discrepancies are due to differences in strain rates), whereas the discrepancy is significant for Al-7075.

These properties were extrapolated to a higher strain rate of $10^{6} \mathrm{~s}^{-1}$ by using the JC plasticity model given by Eq. (1) for characterizing the cavitation pitting behavior. Strain rate sensitivity parameters of the materials $(C)$ already estimated from compression and SHPB tests were used for the extrapolation. Figure 6 shows the nanoindentation stress-strain curves extrapolated to a strain rate of $10^{6} \mathrm{~s}^{-1}$ for all three materials.

In this paper, the material properties were characterized in two different ways and in both the cases the strain rate sensitivity was estimated by compression tests and split Hopkinson Pressure bar tests. The influence of these two approaches on the estimated hydrodynamic impact parameters is now analyzed carefully (in Sec. III) in order to find out the most reliable way of characterizing the material behavior for cavitation pitting.

\section{RESULTS}

\section{A. Estimation of hydrodynamic impact loads}

Cavitation pitting tests were done on all three materials at a flow pressure of 10, 20, and 40 bars for a particular

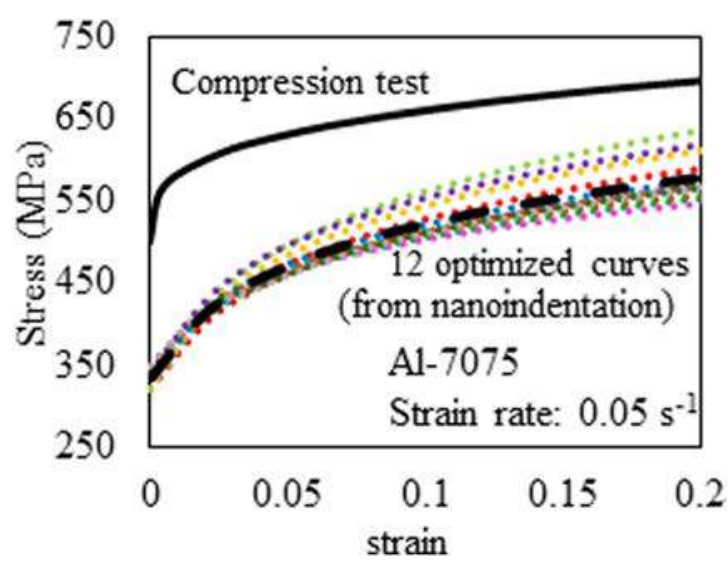

FIG. 5. Compression stress-strain curves at strain rate $0.05 \mathrm{~s}^{-1}$. The 12 optimized curves correspond to nanoindentation stress-strain curves for which the errors between simulated and experimental load-displacement curves were $<8 \mathrm{mN}$.
TABLE II. Nanoindentation material properties at strain rate $0.05 \mathrm{~s}^{-1}$.

\begin{tabular}{lccc}
\hline \hline Material & $\sigma_{y}(\mathrm{MPa})$ & $K(\mathrm{MPa})$ & $n$ \\
\hline Al-7075 & 335 & 396 & 0.3 \\
A-2205 & 508 & 832 & 0.51 \\
NAB & 300 & 1150 & 0.58 \\
\hline \hline
\end{tabular}

duration of time within their incubation period. All the tests were done in a high speed cavitation tunnel that produces geometrically similar flows. Details about the test sample preparation, experimental setup, testing methods, and data analysis technique can be found in Refs. 17 and 18. The test specimen surface was mirror polished prior to the test. After the pitting test is performed the pit dimensions (especially depth and diameter) were estimated by using a contact profilometer with a microprobe of radius $2 \mu \mathrm{m}$. The use of such contact microprobe leads to some error; however, since the pit diameter is significantly larger than the pit depth (see Figure 7) such error is negligible here. For a more accurate measurement, however, a more-advanced technique such as optical or laser profilometry, scanning electron microscopy, or atomic force microscopy could be used. The hydrodynamic impact load parameters, peak stresses $\sigma_{H}$ and their radial extent $d_{H}$ corresponding to all experimental pits, have been estimated using the inverse FE method as presented in Ref. 10. As an example, Figure 7 shows the experimental pits (circular points, for A-2205 material, pitting tested at 40 bars) that were numerically reproduced (square points) by the inverse FE method. On average, 3 simulations were required to get the optimum solution with a maximum error of $1.5 \mu \mathrm{m}$ in pit diameter and $0.05 \mu \mathrm{m}$ in pit depth. Figure 8 shows the distribution of the hydrodynamic impact loads captured by the three materials at 40 bars. As can be seen, the different materials depending on their strength capture different impacts. A-2205 has the maximum strength and captures the impacts of higher magnitudes. The data in Figure 8 are obtained by using the material properties obtained by nanoindentation tests that were extrapolated to a strain rate $10^{6} \mathrm{~s}^{-1}$ (see Figure 6). One important point to be noticed in Figure 8 is that as the impact diameter decreases

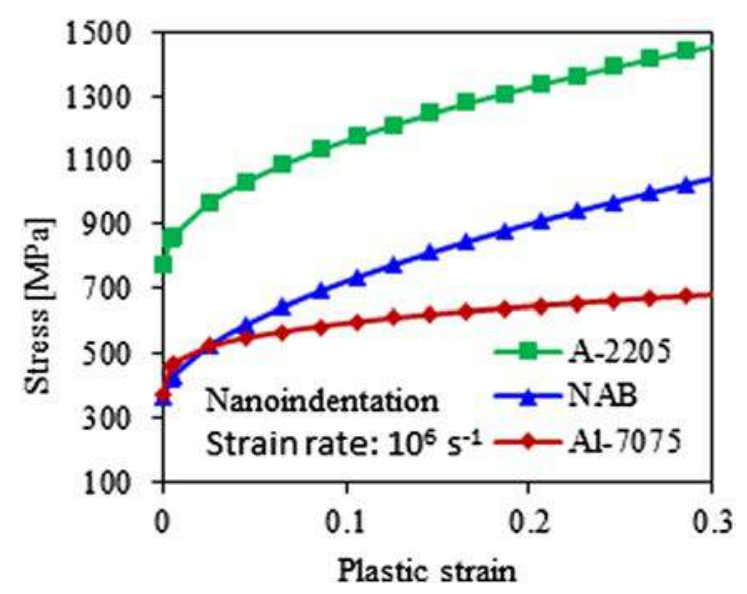

FIG. 6. Stress-strain curve obtained from the nanoindentation tests extrapolated to a strain rate of $10^{6} \mathrm{~s}^{-1}$. 


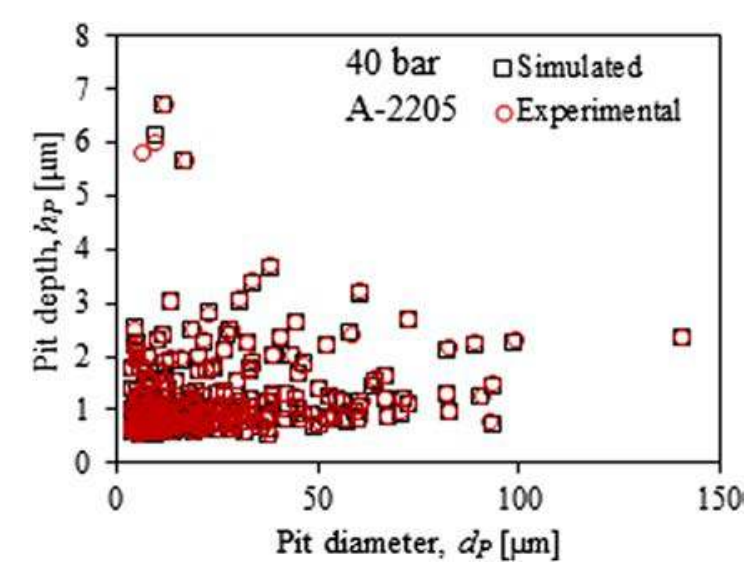

FIG. 7. Experimentally obtained 289 cavitation pits on A-2205 tested at 40 bars. Simulated data represent the pit dimensions when the corresponding experimental pits were optimized by the inverse FE method.

the impact stress increases. This phenomenon is related to the critical pressure needed to collapse a bubble. For large bubbles, the critical pressure is close to the vapor pressure of the liquid. When the bubble size decreases, the surface energy increases and the critical pressure decreases. ${ }^{28}$ For a cavitation bubble to collapse, the mean pressure of the liquid has to drop below the critical pressure. Thus the collapse driving pressure gradient for a smaller bubble is high compared to that of a bigger bubble which is confirmed by the numerical estimations of the impact stresses. ${ }^{14}$

Comparing nanoindentation simulations with cavitation pits, Carnelli et al. ${ }^{20}$ have estimated the strain induced in the material by the impact load required to form a cavitation pit of a given depth and diameter. Then using materials constitutive stress-strain relationship, they could estimate an equivalent stress which they refer to as impact stress. Their method is based on Tabor's equation of strain $^{29}$ which they modified by assuming each cavitation pit as a spherical cap of the measured depth and diameter. Using their method, they could only estimate $\sigma_{H}$, not $d_{H}$, which is also required for better characterization of the cavitating flow. Moreover, it is not clear if the stress calculated into the material could be used as a measure of the impact stress, because earlier in

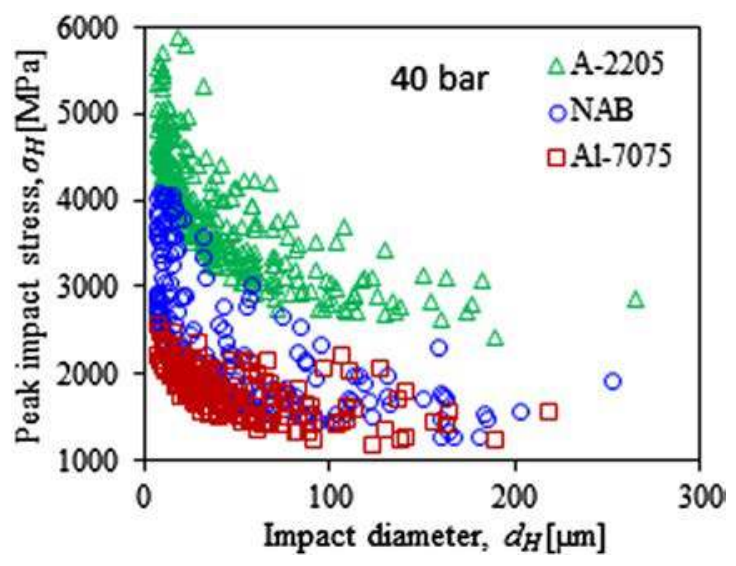

FIG. 8. Distribution of the hydrodynamic impact loads obtained by the inverse FE method at 40 bars of flow pressure. The impact loads for 650 pits have been shown here.
Ref. 10 we have seen that there is a scaling down of the stress from the hydrodynamic impact to the material. To deform plastically a material, the required stresses were a few times higher than the yield strength, which could be due to triaxility.

The aim here is to find out whether the target material itself can be used as a pressure sensor in cavitation pitting or not. If yes, then the question is-how to validate the estimated impact stresses, because as mentioned earlier it is not possible to accurately measure them experimentally. This is a new approach where the authors are trying to use different materials under the same flow condition to get material independent features which would characterize the flow condition only.

In a cavitating flow there would be a large number of impacts of different peak stresses and radial extents. Hence, to characterize the flow the number of impacts per unit area and per unit time for a given range of peak stress $\sigma_{H}$ and radial extent $d_{H}$ have been analyzed. This quantity is termed in this paper as the "normalized impact frequency," $N$ (whose unit is impacts $/ \mathrm{cm}^{2} / \mathrm{s} / \mu \mathrm{m} / \mathrm{MPa}$ ).

The impact load parameters $\sigma_{H}$ and $d_{H}$ have been estimated by the inverse FE method using the compressive material properties (Figure 3) extrapolated to the strain rate of $10^{6} \mathrm{~s}^{-1}$. The normalized impact frequency $N$ is plotted as a function of $\sigma_{H}$ as shown in Figure 9, where $N$ is estimated for different ranges of $\sigma_{H}$ (with a band width of $200 \mathrm{MPa}$ ) and for a given range of $d_{H}=20-40 \mu \mathrm{m}$. As can be seen, the data from the three materials do not follow a single trend, although all the tests were done at the same flow condition of 20 bars. A similar inconsistency was found at other flow conditions of 10 and 40 bars as well, and also for other values of $d_{H}$.

Now, by using the nanoindentation material properties extrapolated to a strain rate of $10^{6} \mathrm{~s}^{-1}$ as shown in Figure 6, the impact load parameters $\sigma_{H}$ and $d_{H}$ have been estimated by the inverse FE method. As before, the normalized impact frequency $N$ is plotted as a function of $\sigma_{H}$ for two different

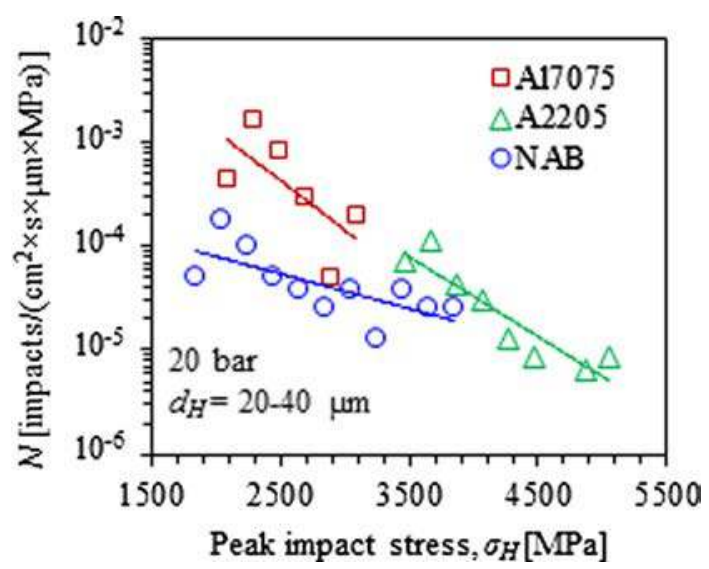

FIG. 9. Normalized impact frequency, $N$ (Number of impacts $/ \mathrm{cm}^{2} / \mathrm{s} / \mu \mathrm{m} /$ $\mathrm{MPa}$ ) is plotted as a function of peak impact stress $\sigma_{H}$ for the hydrodynamic impact loads estimated at 20 bars. $N$ is estimated at different ranges in the values of $\sigma_{H}$ (with a band width of $200 \mathrm{MPa}$ ) for a given range of $d_{H}=20-40 \mu \mathrm{m}$. The vertical axis is on log-scale. Compressive material properties extrapolated at a strain rate $10^{6} \mathrm{~s}^{-1}$ were used for FE simulations in the inverse method. 
flow conditions of 10 and 40 bars, as shown in Figure 10. As can be seen in both the flow conditions, the normalized impact frequency $N$ follows a unique trend in a semi-log plot (vertical axis on log-scale) irrespective of the material being used to capture them. A similar consistency is also found at a flow condition of 20 bars upstream pressure, as well as for other values of $d_{H}$. This is a very conclusive result as it validates the applicability of a target material itself as a pressure sensor. We can also conclude that the impact frequency follows an exponential behavior with the impact stress. Note that a similar exponential behavior of impact load measured experimentally by using a pressure transducer is reported by other authors as well. ${ }^{5}$ The frequency of impact stress increases exponentially as the peak stress decreases. Moreover, as expected, the impact frequency also increases significantly with the flow pressure. Each material depending on its strength filters the hydrodynamic impacts, or in other words, provides the measurements of peak stresses within a certain range. It should be emphasized here that although we have used a single value of strain rate of $10^{6} \mathrm{~s}^{-1}$ in the inverse FE method to estimate the impact load parameters, in reality the strain rate would be different depending on the bubble radius, pressure gradient, and standoff distance. ${ }^{14}$ However, as we have chosen a narrow band width of $d_{H}=20-40 \mu \mathrm{m}$ in Figure 10 , the unique trend indicates the dynamic influence of the strain rate is not a critical issue here for such a statistical analysis.

We can expect some increase in the peak impact stresses with the flow pressure, but as the materials are identical we cannot capture those impacts of higher magnitudes whose frequency of occurrence is less. In order to capture them, a pitting test should be carried out for a longer period of time but, by the mean time the material would erode out because of the repeated impacts of comparatively lower magnitudes. The only way to capture those impacts would be to use another material of even higher strength where impacts of

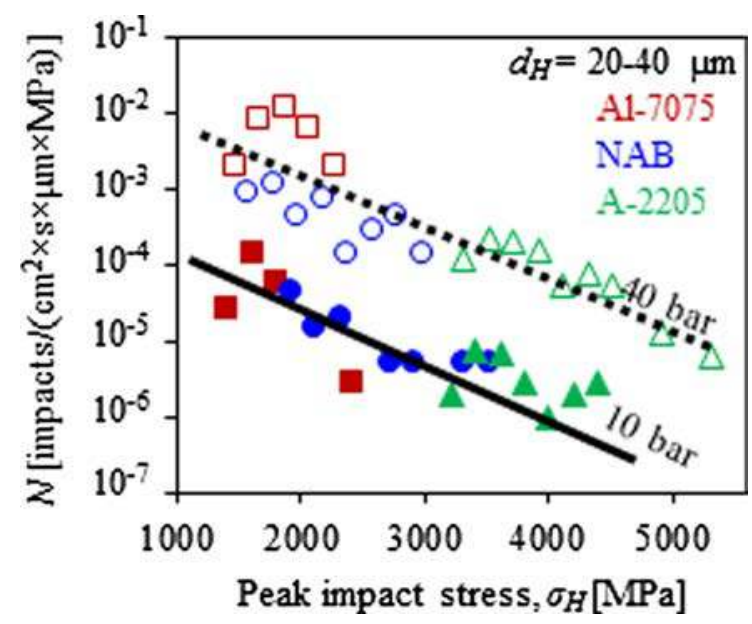

FIG. 10. Normalized impact frequency, $N$ (Number of impacts $/ \mathrm{cm}^{2} / \mathrm{s} / \mu \mathrm{m} /$ $\mathrm{MPa})$ plotted as a function of the peak impact stress $\sigma_{H}$ for the hydrodynamic impact loads estimated at 10 and 40 bars. $N$ is estimated at different ranges in the values of $\sigma_{H}$ (with a band width of $200 \mathrm{MPa}$ ) for a given range of $d_{H}=20-40 \mu \mathrm{m}$. The vertical axis is on log-scale. Nanoindentation material properties extrapolated at a strain rate $10^{6} \mathrm{~s}^{-1}$ were used for the FE simulations in the inverse method. comparatively lower magnitude would not be able to produce any cavitation pit.

In Figure $11, N$ is plotted as a function of $d_{H}$ for two different flow conditions of 10 and 40 bars. Here $N$ is estimated at different ranges in the values of $d_{H}$ (with a band width of $10 \mu \mathrm{m}$ ) for a given range of $\sigma_{H}=2400-2600 \mathrm{MPa}$. As can be seen, it is very difficult to capture impacts of similar magnitudes by using different materials as a sensor as their strengths are different. But all three materials may capture impacts of similar sizes $\left(d_{H}\right)$ as shown in Figure 11. The impact frequency $N$ is found to follow an exponential behavior with $d_{H}$, as the semi-log plots of $N$ vs. $d_{H}$ in Figure 11 are linear. As expected the frequency of impacts for a given $d_{H}$ increases with the flow pressure.

Finally, the flow aggressiveness for a given flow condition can be characterized by a 3D plot as shown in Figure 12 , where the impact frequency $N$ is plotted as a function of $\sigma_{H}$ and $d_{H}$. Values of $N$ are estimated at different points using a grid of $200 \mathrm{MPa} \times 20 \mu \mathrm{m}$ size in $\sigma_{H}-d_{H}$ space. It is observed that all the data points in Figure 12 fit reasonably well the following analytical expression given by Eq. (4)

$$
N=N^{*} \exp \left(-\left(\frac{\sigma_{H}}{\sigma_{H}^{*}}\right)\right) \exp \left(-\left(\frac{d_{H}}{d_{H}^{*}}\right)\right) .
$$

Here the characteristic impact frequency $N^{*}$, characteristic peak impact stress $\sigma_{H}^{*}$, and characteristic impact diameter $d_{H}^{*}$ are three fitting constants which characterize the flow. The $\sigma_{H}^{*}$ represents a mean value of $\sigma_{H}$ over all values of $d_{H}$ and similarly the $d_{H}^{*}$ represents a mean value of $d_{H}$ over all values of $\sigma_{H}$. Estimated values of $N^{*}, \sigma_{H}^{*}$, and $d_{H}^{*}$ at different flow pressures is given in Table III. In order to get a more accurate estimation of $N^{*}, \sigma_{H}^{*}$, and $d_{H}^{*}$ more data are required to reduce the scatter. These parameters depend on the flow condition only, irrespective of the material being used to capture them.

This is a significant achievement as we could explain the flow aggressiveness by an analytical equation of a simple

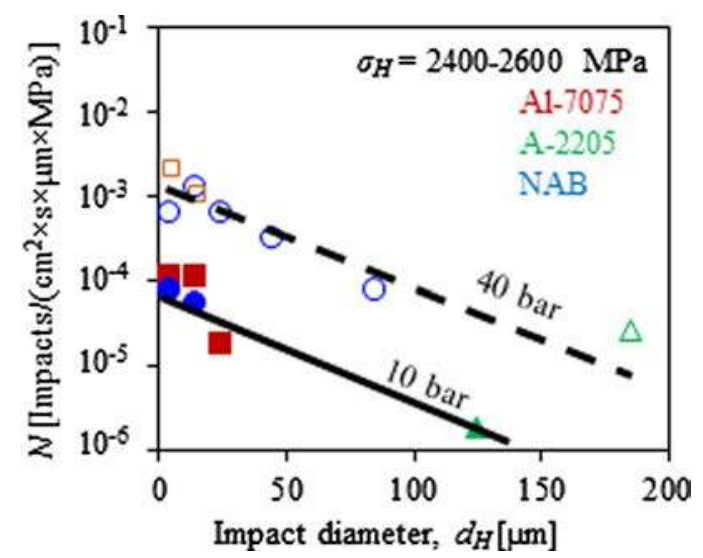

FIG. 11. Normalized impact frequency, $N$ (Number of impacts $/ \mathrm{cm}^{2} / \mathrm{s} / \mu \mathrm{m} /$ $\mathrm{MPa})$ plotted as a function of the impact diameter $d_{H}$ for the hydrodynamic impact loads estimated at 10 and 40 bars. $N$ is estimated at different ranges in the values of $d_{H}$ (with a band width of $10 \mu \mathrm{m}$ ) for a given range of $\sigma_{H}=2400-2600 \mathrm{MPa}$. The vertical axis is on log-scale. Nanoindentation material properties extrapolated at a strain rate $10^{6} \mathrm{~s}^{-1}$ were used for FE simulations in the inverse method. 


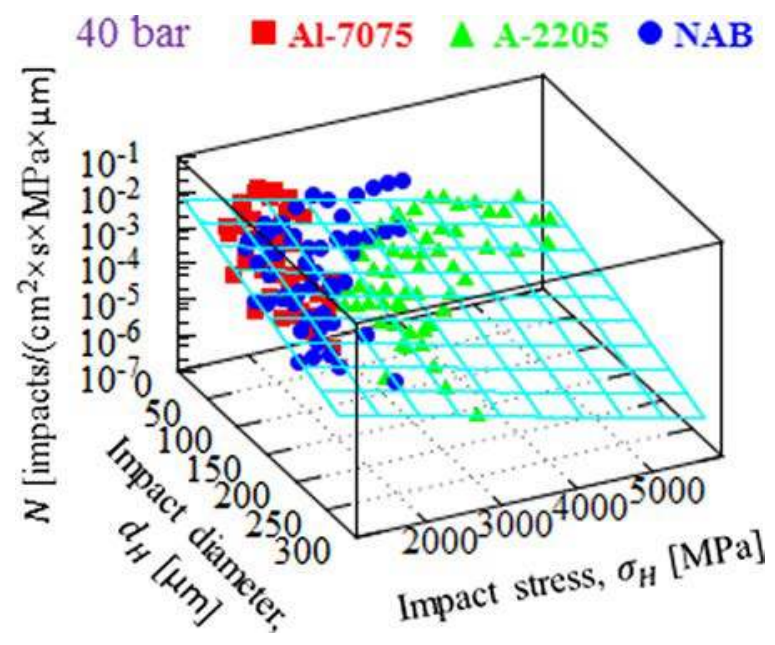

FIG. 12. Plot of the normalized impact frequency, $N$ as a function of the peak impact stress $\sigma_{\mathrm{H}}$ and impact diameter $d_{H}$ for the hydrodynamic impact loads estimated at 40 bars. $N$ is estimated at different grids of $200 \mathrm{MPa} \times 20 \mu \mathrm{m}$ size in $\sigma_{H}-d_{H}$ space. The vertical axis is on log-scale. A total of 650 pits have been analyzed. Nanoindentation material properties extrapolated to the strain rate of $10^{6} \mathrm{~s}^{-1}$ were used for the FE simulations in the inverse method.

exponential form. This would enable us to predict the flow aggressiveness at different flow pressures if we analyze the behavior of $N^{*}, \sigma_{H}^{*}$, and $d_{H}^{*}$ with respect to flow pressure. Doing that would require a very large number of pit data for the statistical analysis. We should also be able to capture the impacts of smaller size with high peak pressure and bigger size with a comparatively low peak pressure by additional cavitation pitting tests, which would be difficult as the overlapping of pits would become unavoidable. So, no further analysis is done in that direction in this paper. It should be mentioned that, though the errors are significant in Table III, when the flow pressure increases, $N^{*}$ increases significantly, whereas $\sigma_{H}^{*}$ and $d_{H}^{*}$ remains almost constant. This is interesting as the cavitation pitting tests at different flow pressures were done with a constant cavitation number ${ }^{17}$ that essentially generates geometrically similar flows, the flow aggressiveness seems to change with the flow pressure in terms of their characteristic frequency only, whereas $\sigma_{H}^{*}$ and $d_{H}^{*}$ seem to be not only material independent but flow independent as well.

\section{DISCUSSION}

As presented in Sec. III, by using the material properties obtained by compression tests extrapolated to a strain rate of $10^{6} \mathrm{~s}^{-1}$, we did not get a unique trend in the plot of $N$ vs. $\sigma_{H}$ as shown in Figure 9, whereas the properties obtained by the nanoindentation tests extrapolated to the same strain rate gave us a unique trend as shown in Figure 10. This unique

TABLE III. Values of $N^{*}, \sigma_{H}^{*}$ and $d_{H}^{*}$ at different flow pressures.

\begin{tabular}{lrcc}
\hline \hline Flow pressure (bars) & \multicolumn{1}{c}{$\sigma_{H}^{*}(\mathrm{MPa})$} & $d_{H}^{*}(\mu \mathrm{m})$ & $N^{*}$ \\
\hline 10 & $1000 \pm 247$ & $50.00 \pm 10$ & $0.0009 \pm 0.0005$ \\
20 & $575 \pm 104$ & $57.54 \pm 10$ & $0.0195 \pm 0.0125$ \\
40 & $890.0 \pm 164$ & $56.54 \pm 10$ & $0.0376 \pm 0.0169$ \\
\hline \hline
\end{tabular}

trend was mandatory to conclude that the target material itself can be used as a sensor in cavitation pitting.

As discussed in Sec. II, it was found that the deformation behavior of Al-7075 in compression and nanoindentation were different, whereas for both A-2205 and NAB compression and nanoindentation behaviors were similar. This difference in the behavior of Al-7075 probably led to the inconsistency in Figure 9 and we concluded that nanoindentation is the proper way to characterize the materials constitutive behavior for cavitation pitting. Thus it is necessary to elucidate the reason and hence microstructural analyses were done.

As a nanoindentation test is done in a confined region compared to that of a compression test, distribution of different phases and their strength will decide the local and global behavior of the probed material. Thus attention was paid to reveal the different phases of the materials, rather than the grain boundaries. Samples were prepared similarly as done for nanoindentation with final polishing using colloidal silica of $0.03 \mu \mathrm{m}$ size. If the different phases in the material have a variation in strength they will be polished to different depths producing contrast under optical microscope. Figures 13(a)-13(c) show the binary images of the phase structures of the three materials, on a plane parallel to the cavitation pitting tested surface, obtained by optical microscope (Olympus BX51M).

Figure 13(a) shows the phases in duplex stainless steel (A-2205) which consists of almost $50 \% \delta$-ferrite and $50 \%$ $\gamma$-austenite. ${ }^{30-32}$ As can be seen, austenite is uniformly distributed into the ferrite matrix with different orientations. It is possible to selectively indent the different phases with an indenter of radius $9.46 \mu \mathrm{m}$ (as used in this study), but the responses would not be very different. This is because the region of the material which would effectively resist the deformation could be considered as a semi-sphere of radius $\left(R_{d}\right)$ 2-3 times bigger than the indenter radius $R$ as shown in Figure 14 (obtained by nanoindentation simulation of A-2205 with $R=9.46$ ), and hence this region will always contain both phases. Therefore, the local and global behavior in nanoindentation and compression tests, respectively, would be almost identical for A-2205. Moreover, El Mehtedi et al. $^{31}$ and $\mathrm{Hay}^{32}$ have done nanoindentation tests on the different phases of 2205 duplex stainless steel and found no significant difference in their hardness or Young's modulus. Their findings also support the previous statement. This could be the reason why the FE simulation of nanoindentation using the compression test data for the constitutive equation yielded a load-displacement curve similar to the experimental one as discussed in Sec. II D.

Figure 13(b) shows the different phases in NAB. The microstructure of the material is similar to that observed by several other authors. ${ }^{33-35}$ The commonly observed phases are copper-rich solid solution ( $\alpha$-phase) matrix, different $k$-phases (iron-bearing phases $k_{I}, k_{I I}$ and $k_{I V}$ are based on $\mathrm{Fe}_{3} \mathrm{Al}$ and $\mathrm{NiAl}$ based $k_{I I I}$ phase), and some amount of retained- $\beta$-phase which finally converts to martensite. Easily distinguishable phases have been highlighted in Figure 13(b) based on their morphology. As can be seen all the phases are almost uniformly distributed into the matrix material (could 


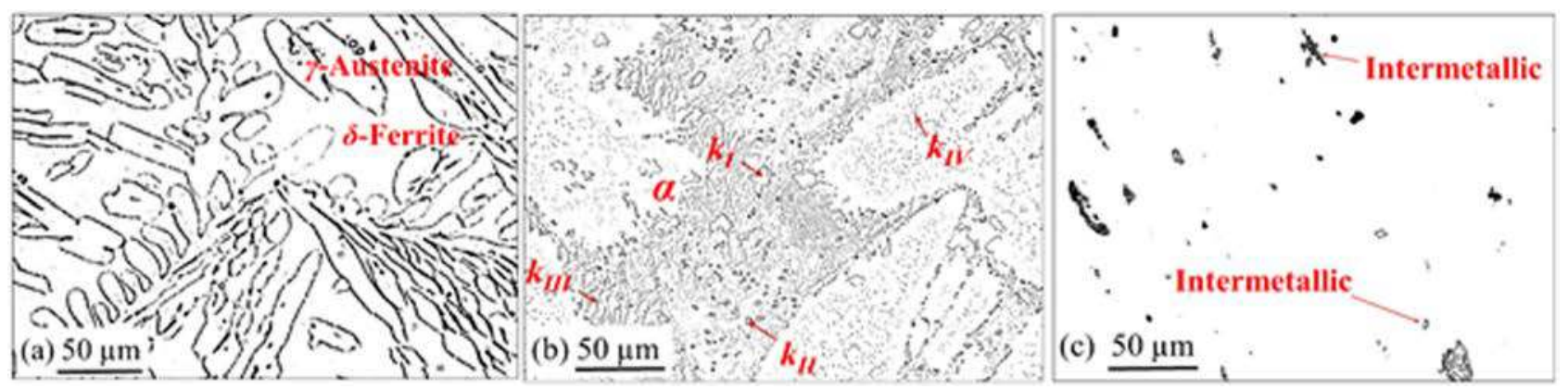

FIG. 13. Phase structures of all three materials obtained by optical microscopy (OLYMPUS BX51M). (a) 2205 duplex stainless-steel (A-2205), (b) NickelAluminum Bronze (NAB), and (c) 7075 Aluminum alloy (Al-7075).

be seen in Refs. 33-35 as well), so for NAB we can expect that the local and global behavior would be similar. This is probably the reason for NAB also, for which the FE simulation of a nanoindentation test gives a load-displacement curve similar to the experimental one when the compression parameters are used in the FE modeling (see Sec. II D).

Figure 13(c) shows the different phases in 7075 aluminum alloy (Al-7075). Many authors ${ }^{36-38}$ have done extensive microstructural analysis on this material, especially regarding the distribution of the second phases into the matrix. The second phases are generally Fe-bearing inclusions $\left(\mathrm{Al}_{7} \mathrm{Cu}_{2} \mathrm{Fe}_{2}, \mathrm{Al}_{23} \mathrm{Fe}_{4} \mathrm{Cu}\right)$ and Si-bearing inclusions $\left(\mathrm{Mg}_{2} \mathrm{Si}\right)$ which are uniformly distributed into the matrix, and their volume fraction could reach up to $3 \%{ }^{36,38}$ Particle size generally varies depending on the manufacturing processes and mechanical treatment applied. Particle size could be as big as $150 \mu \mathrm{m}$ or even more ${ }^{36}$ with even larger inter particle spacing. However, most of the inclusions in the current material have sizes from 10 to $20 \mu \mathrm{m}$ and a maximum particle size of $\sim 50 \mu \mathrm{m}$ is observed, as can be seen in Figure 13(c). Although the volume fraction is less, the uniform distribution of these inclusions throughout the material would influence the mechanical properties of the material. The authors in Ref. 36 have performed nanoindentation tests

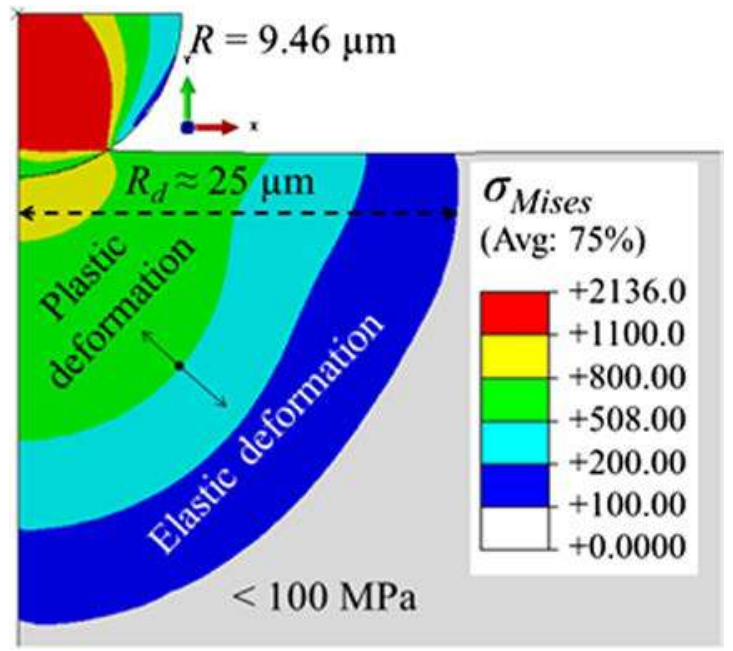

FIG. 14. Nanoindentation simulation of A-2205 with a spherical diamond indenter of radius $R=9.46 \mu \mathrm{m}$. Distribution of von Mises equivalent stress $\left(\sigma_{\text {Mises }}\right)$ is shown highlighting the extent of an effectively deformed region beneath the indenter. on these different phases and have found huge differences in their strength compared to that of the matrix phase. Thus we can expect that in cavitation pitting or in nanoindentation the local behavior of the material would be different from the global or bulk behavior in compression. In compression the behavior would be like a composite material of soft and hard phases, whereas in nanoindentation depending on the phase we might get a strong or weak response. This is probably the reason for which compressive material properties used in the FE simulation of nanoindentation did not give a load-displacement curve similar to the experimental one. So when we optimized the constitutive parameters by the inverse nanoindentation method as discussed in Sec. II D, we got a softer stress-strain response compared to that of the compression test as shown in Figure 6. In the cavitation test, the soft matrix of Al-7075 will erode out preferentially. Therefore to characterize this type of material for cavitation pitting the local behavior of the soft phases should be considered and the nanoindentation test is the preferred option to get the material properties. The above hypothesis on Al7075 is also supported by the findings in Ref. 18 which shows that cavitation erosion resistance (which is related to material strength) of Al-7075 is significantly less compared to that of NAB or A-2205, although the tension or compression strength of Al-7075 is significantly higher than NAB or A-2205.

As presented throughout the paper, the inverse FE method is a potential technique that could be used in practice to estimate the hydrodynamic impact loads in cavitation pitting. Though improvements can be done, especially in terms of dynamic explicit FE analysis, to take into account the effect of inertia of the material and possible change in strain rates for all the hydrodynamic impacts, the method is operational. The dynamic explicit analysis of cavitation pitting performed in Ref. 13 on these same three materials has shown that for impact duration of $1 \mu \mathrm{s}$ or more, the contribution of an inertial effect to the dynamics of cavitation pitting becomes negligible, it is essentially the strain rate sensitivity which influences the pit dimensions depending on the impact duration. Thus, if a material is selected carefully with minimum strain rate sensitivity (like Al-7075), accurate measurements of the hydrodynamic impact loads could be obtained by the inverse FE method using static FE analysis. Obviously, dynamic explicit analysis of the material behavior for the inverse FE method would certainly lead to a more 
accurate estimation of impact loads, but for that the knowledge of impact duration corresponding to each cavitation pit is required. To the best of our knowledge there is no way to estimate impact duration based on cavitation pit dimensions and hence FE static analysis with extrapolated material properties is used here. The main difficulty to implement the method is that this is a time consuming method as it requires several experiments and a thorough analysis for calibration of material properties. Although it is found that the Gaussian profile for the pressure field is closely related to the cavitation impact loading and also used by several other authors, in practice the actual pressure field associated with cavitation bubble collapse has a more complex shape both in space and time, especially due to interaction of collapsing bubbles. ${ }^{14}$ As the method estimates the impact loads from cavitation pit geometries which are all localized plastically deformed regions, brittle material could not be used to estimate the spectra of impact loads. Moreover, if a polymeric material is used to estimate the impact loads, the damping effect which is usually very high compared to the negligible effect in the case of metallic alloy must be considered in the constitutive modeling. Despite all the difficulties, from a statistical point of view the estimated values of the impact loads are quite satisfactory as they agree well with the values in literatures. $^{5,14}$

\section{CONCLUSIONS}

The prime focus of this study was to answer two questions: (1) Can the target material itself be used as a sensor in cavitation pitting to estimate hydrodynamic impact loads, i.e., their peak stress $\sigma_{H}$ and radial extent $d_{\mathrm{H}}$ ? (2) If yes, then how to characterize the material properties or constitutive behavior?

Three materials, Al-7075, A-2205, and NAB, were chosen on which cavitation pitting tests were done at different flow conditions $(10,20$, and 40 bars) and then the resulted pit dimensions (depth, $h_{P}$, and diameter, $d_{P}$ ) were measured. The peak stress $\sigma_{H}$ and radial extent $d_{H}$ of the hydrodynamic impacts corresponding to each pit have been estimated by using an inverse FE method presented in Ref. 10. Quite interestingly a statistical analysis of the estimated impact loads at all the flow conditions was found to be material independent. This is the most important result of this paper which confirms that the target material itself can be used as a pressure sensor.

To answer the second question, material properties required by the inverse FE method were obtained by compression and nanoindentation tests which represent the global and local behavior, respectively. It was found that depending on the microstructure the global and local behavior may vary significantly. Unlike A-2205 and NAB, stress-strain curves for Al-7075 obtained by compression and nanoindentation tests were significantly different. The stress-strain curves were extrapolated to a higher strain rate of $10^{6} \mathrm{~s}^{-1}$, relevant to cavitation pitting. The consistency of impact load spectra between the three materials was obtained using the local material properties obtained by nanoindentation whereas the global properties led to inconsistent results. This is also a very important conclusion which proves that the characterization of the material's local behavior by nanoindentation is relevant to cavitation pitting. In our approach we assumed that the strain rate sensitivity coefficient estimated from split Hopkinson bar tests could be reasonably used for the extrapolation of the nanoindentation data to a strain rate as large as $10^{6} \mathrm{~s}^{-1}$ (which are not accessible in nanoindentation).

Apart from these, it was observed that the flow aggressiveness (which effectively counts the number of impacts for a given range of $\sigma_{H}$ and $d_{\mathrm{H}}$ ) could be represented by a 3D surface, which follows an exponential form as given by Eq. (4). Such a surface can entirely be defined by three parameters, the characteristic impact frequency $N^{*}$, the characteristic peak impact stress $\sigma_{H}^{*}$, and the characteristic impact diameter $d_{H}^{*}$ which are material independent.

On-going and future work is focused on the simulation of multiple impacts where the estimated impact loads $\left(\sigma_{H}\right.$ and $d_{\mathrm{H}}$ ) would be applied repetitively and randomly on the material surface to estimate mass loss with time. Thus the final aim is to numerically predict mass loss evolution during cavitation erosion for which the knowledge of $\sigma_{H}$ and $d_{\mathrm{H}}$ is necessary. For that reason complete dynamic analysis and additional material parameters to account for damage will be considered in the forthcoming FE simulations.

\section{ACKNOWLEDGMENTS}

This research was conducted under the Naval International Cooperative Opportunities in Science and Technology Program (NICOP, Grant No. N62909-12-17112) funded by the Office of Naval Research. The authors wish to thank Dr. Ki-Han Kim from the Office of Naval Research (ONR) and Dr. Woei-Min Lin from the Office of Naval Research Global (ONRG) who supported this work. They are also grateful to Michel Riondet (LEGI) for conducting the cavitation pitting tests and Nicolas Ranc for the compression and Split Hopkinson Pressure Bar tests conducted at PIMM.

${ }^{1}$ N. K. Bourne and J. E. Field, J. Appl. Phys. 78, 4423 (1995).

${ }^{2}$ J.-C. Isselin, A.-P. Alloncle, and M. Autric, J. Appl. Phys. 84, 5766 (1998).

${ }^{3}$ D. Fernandez Rivas, J. Betjes, B. Verhaagen, W. Bouwhuis, T. C. Bor, D. Lohse, and H. J. G. E. Gardeniers, J. Appl. Phys. 113, 064902 (2013).

${ }^{4}$ S. Hattori, T. Hirose, and K. Sugiyama, Wear 269, 507 (2010).

${ }^{5}$ J.-P. Franc, M. Riondet, A. Karimi, and G. L. Chahine, J. Fluids Eng. 133, 121301 (2011).

${ }^{6}$ Y. C. Wang and Y. W. Chen, Exp. Therm. Fluid Sci. 32, 403 (2007).

${ }^{7}$ T. Okada, S. Hattori, and M. Shimizu, Wear 186-187, 437 (1995).

${ }^{8}$ M. S. Mihatsch, S. J. Schmidt, M. Thalhamer, and N. A. Adams, in Proceedings of the 8th International Symposium on Cavitation, Singapore, 13-16 August 2012, pp. 1-6.

${ }^{9}$ S. J. Schmidt, M. S. Mihatsch, M. Thalhamer, and N. A. Adams, in Advanced Experimental and Numerical Techiques for Cavitation Erosion Prediction, Fluid Mechanics and Its Applications Vol. 106, edited by K.-H. Kim, G. Chahine, J. Franc, and A. Karimi (Springer, Netherlands, 2014), pp. 329-344.

${ }^{10}$ S. C. Roy, J. P. Franc, C. Pellone, and M. Fivel, "Determination of cavitation load spectra-Part 1: Static finite element approach," Wear (to be published).

${ }^{11}$ R. T. Knapp, Trans. ASME 77, 1045 (1955).

${ }^{12}$ R. T. Knapp, Trans. Am. Soc. Mech. Eng. 80, 91 (1958). 
${ }^{13}$ S. C. Roy, J.-P. Franc, N. Ranc, and M. Fivel, "Determination of cavitation load spectra-Part 2: Dynamic finite element approach," Wear (to be published).

${ }^{14}$ G. L. Chahine, in Advanced Experimental and Numerical Techniques for Cavitation Erosion Prediction, Fluid Mechanics and Its Applications Vol. 106, edited by K.-H. Kim, G. L. Chahine, J.-P. Franc, and A. Karimi (Springer, 2014), pp. 123-161.

${ }^{15}$ J.-K. Choi, A. Jayaprakash, A. Kapahi, C.-T. Hsiao, and G. L. Chahine, J. Mater. Sci. 49, 3034 (2014).

${ }^{16}$ F. Pöhl, S. Mottyll, R. Skoda, and S. Huth, Wear 330-331, 618 (2015).

${ }^{17}$ J.-P. Franc, J. Fluids Eng. 131, 021303 (2009).

${ }^{18}$ J.-P. Franc, M. Riondet, A. Karimi, and G. L. Chahine, Wear 274-275, 248 (2012)

${ }^{19}$ D. Carnelli, A. Karimi, and J.-P. Franc, J. Mater. Res. 27, 91 (2012).

${ }^{20}$ D. Carnelli, A. Karimi, and J. P. Franc, Wear 289, 104 (2012).

${ }^{21}$ A. Karimi and J. L. Martin, Int. Met. Rev. 31, 1 (1986).

${ }^{22}$ G. R. Johnson and W. H. Cook, Eng. Fract. Mech. 21, 31 (1985).

${ }^{23}$ J. M. Collin, G. Mauvoisin, O. Bartier, R. El Abdi, and P. Pilvin, Mater. Sci. Eng., A 501, 140 (2009).

${ }^{24}$ E. G. Herbert, W. C. Oliver, and G. M. Pharr, Philos. Mag. 86, 5521 (2006).

${ }^{25} \mathrm{G}$. T. Gray, in ASM Handbook, Mechanical Testing and Evaluation Vol. 8 (ASM International, Materials Park, OH, 2000), pp. 1027-1068.

${ }^{26}$ C. Moussa, X. Hernot, O. Bartier, G. Delattre, and G. Mauvoisin, J. Mater. Sci. 49, 592 (2014).
${ }^{27}$ M. Dao, N. Chollacoop, K. J. Van Vliet, T. A. Venkatesh, and S. Suresh, Acta Mater. 49, 3899 (2001).

${ }^{28}$ G. L. Chahine, J.-P. Franc, and A. Karimi, in Advanced Experimental and Numerical Techiques for Cavitation Erosion Prediction, Fluid Mechanics and Its Applications Vol. 106, edited by K.-H. Kim, G. L. Chahine, J.-P. Franc, and A. Karimi (Springer, 2014), pp. 3-20.

${ }^{29} \mathrm{D}$. Tabor, The Hardness of Metals (Clarendon Press, 1951).

${ }^{30}$ C. Herrera, D. Ponge, and D. Raabe, Steel Res. Int. 79, 10 (2008).

${ }^{31}$ M. El Mehtedi, S. Spigarelli, P. Ricci, C. Paternoster, and E. Quadrini, La Metall. Ital. 9, 11 (2010).

${ }^{32}$ J. Hay, "Mapping the mechanical properties of 2205 duplex stainless steel," Agilent Technologies, Application Note, Document No. 59910381EN Review A, 2013.

${ }^{33}$ A. Jahanafrooz, F. Hasan, G. W. Lorimer, and N. Ridley, Metall. Trans. A 14, 1951 (1983).

${ }^{34} \mathrm{~A}$. Al-Hashem and W. Riad, Mater. Charact. 48, 37 (2002).

${ }^{35}$ F. Hasan, A. Jahanafrooz, G. W. Lorimer, and N. Ridley, Metall. Trans. A 13, 1337 (1982).

${ }^{36}$ S. S. Singh, C. Schwartzstein, J. J. Williams, X. Xiao, F. De Carlo, and N. Chawla, J. Alloys Compd. 602, 163 (2014).

${ }^{37}$ Y. Xue, H. El Kadiri, M. F. Horstemeyer, J. B. Jordon, and H. Weiland, Acta Mater. 55, 1975 (2007).

${ }^{38}$ A. D. Rollett, R. Campman, and D. Saylor, Mater. Sci. Forum 519-521, 1 (2006). 